\title{
Direct visualization of concerted proton tunnelling in a water nanocluster
}

\author{
Xiangzhi Meng ${ }^{1 \dagger}$, Jing Guo ${ }^{1 \dagger}$, Jinbo Peng ${ }^{1 \dagger}$, Ji Chen ${ }^{1}$, Zhichang Wang ${ }^{1}$, Jun-Ren Shi ${ }^{1,2}$, Xin-Zheng Li ${ }^{3,2}$, \\ En-Ge Wang ${ }^{1,2 \star}$ and Ying Jiang ${ }^{1,2 \star}$
}

\begin{abstract}
Proton transfer through hydrogen bonds plays a fundamental role in many physical, chemical and biological processes ${ }^{1-5}$. Proton dynamics is susceptible to quantum tunnelling, which typically involves many hydrogen bonds simultaneously, leading to correlated many-body tunnelling ${ }^{6-9}$. In contrast to the well-studied incoherent single-particle tunnelling, our understanding of many-body tunnelling is still in its infancy. Here we report the real-space observation of concerted proton tunnelling in a cyclic water tetramer using a cryogenic scanning tunnelling microscope. This is achieved by monitoring the reversible interconversion of the hydrogen-bonding chirality of the water tetramer with a chlorine-terminated scanning tunnelling microscope tip. We found that the presence of the $\mathrm{Cl}$ anion at the tip apex may either enhance or suppress the concerted tunnelling process, depending on the details of the coupling symmetry between the $\mathrm{Cl}$ ion and the protons. Our work opens up the possibility of controlling the quantum states of protons with atomic-scale precision.
\end{abstract}

Many-body correlated tunnelling of protons is extremely sensitive to coupling with the atomic-scale environment owing to the demanding phase coherence between the protons. Spectroscopic techniques are not able to provide such local information because of the limitation of spatial resolution ${ }^{5-7,10,11}$. Recently, the scanning tunnelling microscope (STM) has proved to be ideal for probing the intramolecular and intermolecular proton dynamics at the singlemolecule level ${ }^{12-16}$. However, most of these studies are focused on classical over-barrier proton hopping, whereas through-barrier quantum tunnelling of protons has yet to be explored. Furthermore, tracking proton motion along hydrogen bonds requires the ability to resolve the hydrogen-bonding directionality ${ }^{17}$. Although it was recently demonstrated that non-contact atomic force microscopy (NC-AFM; ref. 18) and state-of-the-art STM-based techniques ${ }^{19}$ are able to visualize individual hydrogen bonds with unprecedented resolution, the asymmetry of the hydrogen bond and the extent of quantum delocalization of the shared proton has not been directly distinguishable.

Here we show the possibility of directly visualizing the concerted tunnelling of four protons in an individual hydrogen-bonded water tetramer adsorbed on a Au-supported $\mathrm{NaCl}(001)$ film. This is achieved by monitoring the reversible interconversion of the hydrogen-bonding directionality and associated chirality of the tetramer using a submolecular orbital-imaging technique ${ }^{20}$. To investigate the effect of the atomic-scale environment on the tunnelling process in a well-controlled manner, we functionalize the STM tip apex with a single chlorine anion and then tune the $\mathrm{Cl}$-proton electric coupling in three dimensions with picometre precision. We found that symmetric coupling between the $\mathrm{Cl}$ anion and the four protons considerably enhances the tunnelling probability, whereas asymmetric coupling easily destroys the quantum coherence and the cooperativity of the four protons, resulting in rapid quenching of the concerted tunnelling. Our experimental results are corroborated by density functional theory (DFT) calculations.

Water tetramers are constructed by manipulating four individual $\mathrm{H}_{2} \mathrm{O}$ monomers on a $\mathrm{NaCl}(001)$ bilayer film at $5 \mathrm{~K}$ (see Methods). Two different chiral states of the tetramers can be clearly distinguished from submolecular-resolution STM images (see inset of Fig. 1b), which are attributed to the highest occupied molecular orbital (HOMO) of adsorbed water in our previous work ${ }^{20}$. These two chiral states are in perfect registry with the $\mathrm{NaCl}(001)$ surface, and thus almost perfectly degenerate. Apparently, the chirality of the tetramer could be switched if the four hydrogen-bonded protons collectively hop towards the neighbouring $\mathrm{O}$ atoms through the hydrogen bonds. To investigate the effect of an atomic-scale environment (such as ions) on the proton dynamics, in a controlled manner, we functionalize the tip apex with a single chlorine atom (see Supplementary Fig. 1 and Movie 1), which can provide a long-range electrical interaction with the protons owing to its electronegative nature (Fig. 1a).

The switching dynamics of the tetramer chirality can be monitored by recording the tunnelling current as a function of time (Fig. 1b). The Cl-terminated tip is positioned slightly off the centre of a clockwise tetramer (marked as green stars in the inset of Fig. 1b) to read out the current difference between the two chiral states. When the tip is far above the tetramer, the current remains constant, suggesting no switching between the two chiral states. Once the tip height is reduced by about $230 \mathrm{pm}$, two current levels emerge, in addition to a sudden increase of the tunnelling current. We can retract the tip to the initial height at any of the two levels and rescan the tetramer to check the resulting chirality. The results unambiguously show that the low and high current levels correspond to the clockwise state (CS) and the anticlockwise state (AS) of the tetramer, respectively (see Supplementary Movie 2). More than two current levels occasionally emerged in the current trace during chirality switching owing to the instability of the $\mathrm{Cl}$ atom at the tip apex (Supplementary Fig. 2).

To explore the mechanism of the proton transfer in the tetramer, the switching rates of $\mathrm{CS} \rightarrow$ AS and AS $\rightarrow$ CS are extracted from the current versus time trace (Supplementary Fig. 3). As shown in Fig. 2a, both switching rates are independent of the magnitude as well as the polarity of the sample bias and the tunnelling current. It is worth noting that the switching does not cease, even near

\footnotetext{
${ }^{1}$ International Center for Quantum Materials, School of Physics, Peking University, Beijing 100871, China. ${ }^{2}$ Collaborative Innovation Center of Quantum Matter, Beijing 100871, China. ${ }^{3}$ School of Physics, Peking University, Beijing 100871, China. †These authors contributed equally to this work.

*e-mail: egwang@pku.edu.cn; yjiang@pku.edu.cn
} 

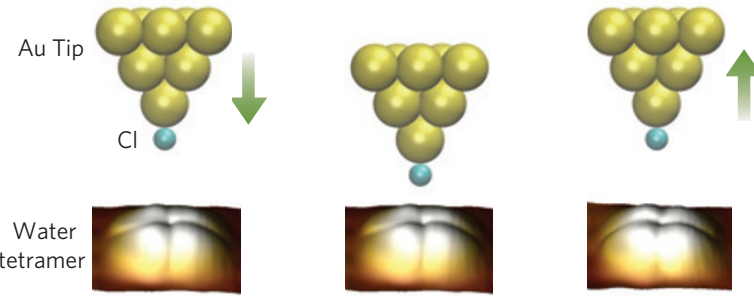

b

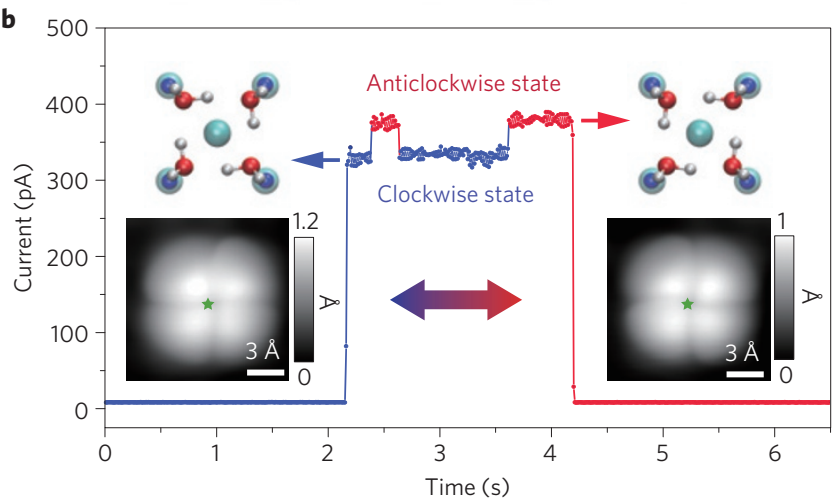

Figure 1 | Chirality switching of a $\mathrm{H}_{2} \mathrm{O}$ tetramer. a, Schematic showing manipulation of the chirality of the tetramer by a Cl-terminated tip. Left: the tetramer stays in the clockwise state (CS) when the tip is far away from the tetramer (gap set with $V=5 \mathrm{mV}$ and $I=5 \mathrm{pA}$ ). Middle: reducing the tip height by $230 \mathrm{pm}$ leads to chirality switching. Right: lifting the tip back to the initial height leaves the tetramer in the anticlockwise state (AS).

b. Tunnelling current trace recorded during the chirality manipulation shown in a. Two current levels can be clearly distinguished in the range 300-400 pA, where the low and high current levels correspond to CS and $A S$, respectively. Left and right insets: Adsorption configuration (upper) and STM images (lower) of CS and AS tetramers, respectively. Parameters for the STM images: $V=20 \mathrm{mV}$ and $I=150 \mathrm{pA}$. The green stars in the STM images denote the tip position where the current trace is acquired. $\mathrm{O}, \mathrm{H}$, $\mathrm{Au}, \mathrm{Cl}^{-}$and $\mathrm{Na}^{+}$are denoted by red, white, golden, cyan and blue spheres, respectively.

zero bias. Those facts reveal that the proton transfer does not result from the excitation of the inelastic tunnelling electrons ${ }^{21}$, nor is it driven by the applied electric field between the tip and the sample ${ }^{22}$. The switching rates also show a very weak dependence on temperature over the range of experimental conditions used (Fig. 2b), clearly demonstrating that the dynamic behaviour of the tetramer is non-Arrhenius in character, and thus not dominated by thermal fluctuations ${ }^{23}$. Therefore, we attribute the interconversion between the two chiral states to quantum tunnelling of protons between the water molecules. Further evidence of proton tunnelling comes from the isotope-exchange experiments, where the switching rates drop by at least two orders of magnitude on the substitution of hydrogen by deuterium (Supplementary Fig. 4).

The proton tunnelling in the tetramer shows a distinct dependence on the tip height (Fig. 2c). As the tip approaches the tetramer, both switching rates (CS $\rightarrow$ AS and AS $\rightarrow$ CS) exhibit an initial rise (region I) followed by a rapid drop (region II). In addition, we notice that the two switching rates gradually deviate from each other as the tip is lowered, which implies that CS and AS may have different energies in the presence of the tip ${ }^{16}$. This energy asymmetry can be understood by considering that the tip is not positioned at the highsymmetry point of the tetramer to read out the current difference (see the inset STM images of Fig. 1b). The tip-induced asymmetry of the double-well potential will open a dissipative channel of the phonon bath, leading to incoherent tunnelling ${ }^{24}$.

To understand the dependence of the switching rates on the tip height, we carried out $a b$ initio DFT calculations using the climbing
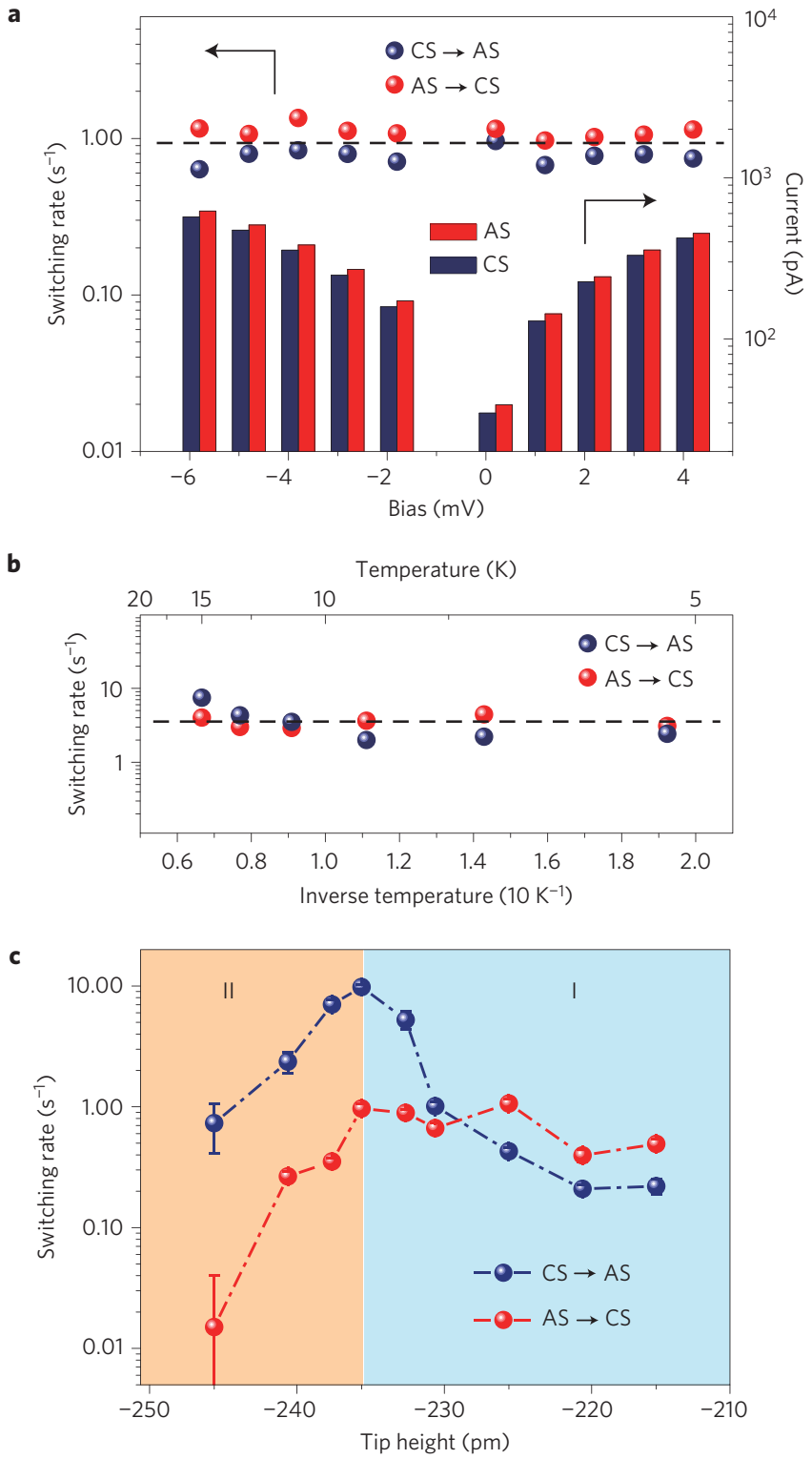

Figure 2 | Quantitative analysis of the switching rate. a, Switching rate and tunnelling current as functions of the sample bias. The tip height is fixed at $-295 \mathrm{pm}$. b. Dependence of the switching rate on temperature. Tip height: $-260 \mathrm{pm}$, sample bias: $5 \mathrm{mV}$. In $\mathbf{a}$ and $\mathbf{b}$ the statistical error is smaller than the data markers; the average of the switching rates for different bias and temperature is denoted by horizontal dashed lines, showing the negligible dependence on those factors. $\mathbf{c}$, Dependence of the switching rate on tip height. Sample bias: $5 \mathrm{mV}$. The switching rates are sensitive to the tip height, exhibiting an initial rise (region I) followed by a rapid drop (region II) with decreasing tip height. The error bar represents the standard error. The tip height is referenced to the gap set with $V=5 \mathrm{mV}$ and $I=5 \mathrm{pA}$.

image nudged elastic band (cNEB; see Methods for details) method. We found that the barrier height is its lowest when the four protons concertedly hop from the hydrogen-bond donor to the acceptor (see the black curve in Fig. 3a). Any conceivable sequential or stepwise rearrangement of the protons would result in significantly higher barrier heights (Supplementary Fig. 5). When a Cl-terminated tip is positioned above the centre of the tetramer, the reaction barrier is effectively suppressed, both in height and width (see the red curve in Fig. 3a).

After taking into account the zero-point energy difference between the initial and transition states (see Methods for details), 

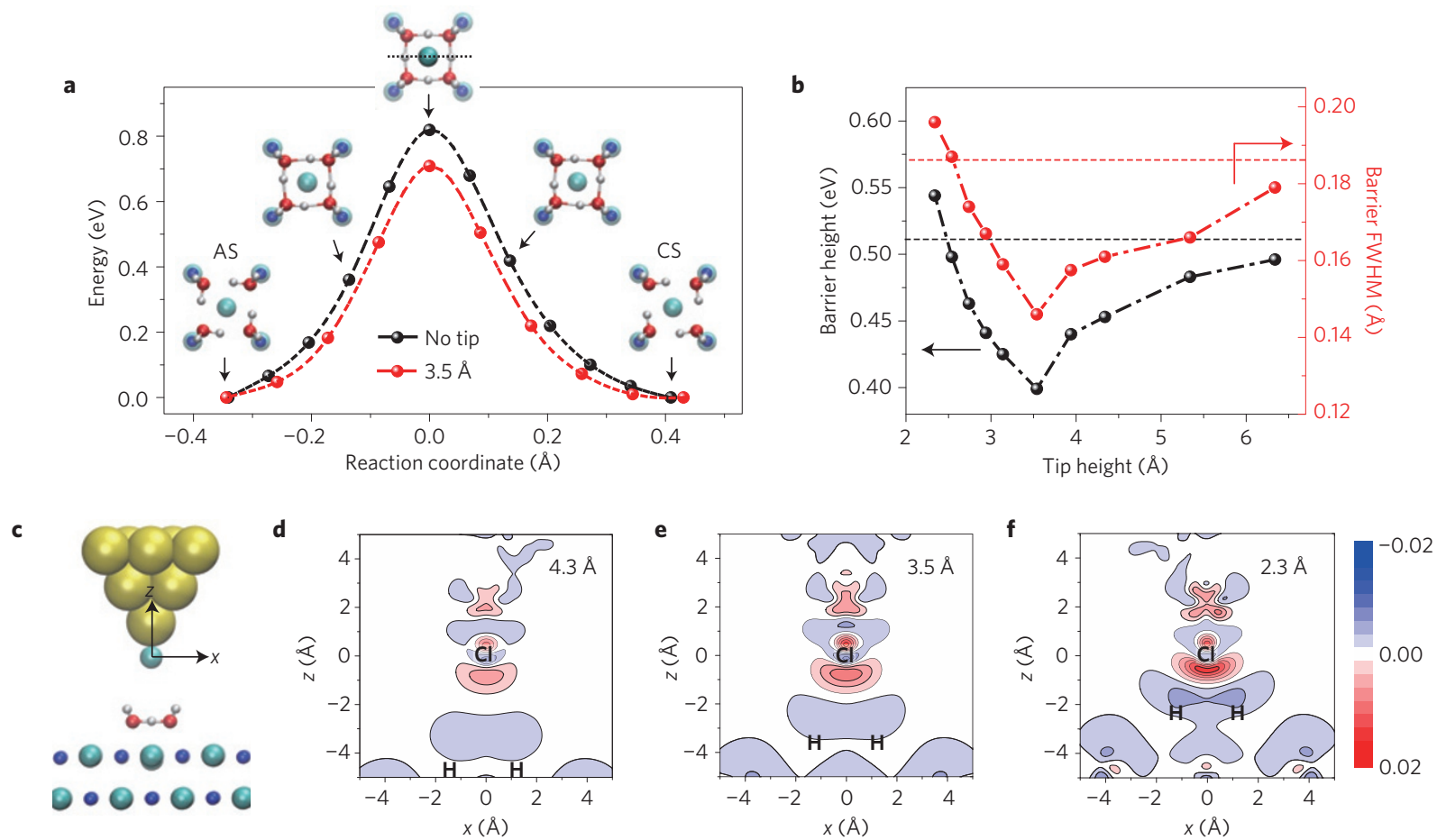

Figure 3 | Effect of the tip on the reaction barrier for proton transfer. a, Reaction barrier for interconversion between CS and AS of the tetramer without (black) and with a Cl-terminated tip at a height of $3.5 \AA$ (red), calculated using the cNEB method. Insets show snapshots along the transition path. $\mathbf{b}$, Plots of the effective barrier height and full-width at half-maximum (FWHM) as a function of tip height. The tip height is defined as the distance between the $\mathrm{Cl}$ atom on the tip and the centroid of four oxygen atoms of the water tetramer. The horizontal black and red dashed lines denote the barrier height and width without the tip, respectively. $\mathbf{c}$, Atomic model of the $\mathrm{Cl}$-tip/tetramer/ $\mathrm{NaCl}$-bilayer system. The origins of $x$ and $z$ axis are set at the $\mathrm{Cl}$ atom on the tip. d-f, Two-dimensional slices of the electron density difference when a Cl tip is placed above the tetramer at $4.3 \AA$ (d), $3.5 \AA$ (e) and $2.3 \AA$ (f). The electron density difference is plotted in a plane perpendicular to the surface, which is marked by a dotted line in the uppermost snapshot of a. Red and blue in the colour bar represent electron gain and depletion, respectively. The units of electron density are $e \AA^{-3}$.

we can obtain the effective barrier height and width, which keep decreasing until the tip height is reduced to about $3.5 \AA$ (Fig. 3b). Further approaching the tip results in a rapid increase of the barrier height and width. We notice that tip-induced rearrangements of the molecules, most probably the simultaneous rigid rotation of the four water molecules, can also lead to interconversion between CS and AS. However, the calculated barrier height and width of this reaction pathway exhibit a monotonic decrease when lowering the tip (Supplementary Fig. 6), which is not consistent with the experimental observation (Fig. 2c).

The minimal reaction barrier occurs for a tip height of roughly $3.5 \AA$, which is still too high to be overcome by classical thermal fluctuations or hot-electron injection under our experimental conditions $(T<20 \mathrm{~K}$ and $V<10 \mathrm{mV})$, thus confirming that the proton transfer can take place only through quantum tunnelling. In fact, the concerted proton motion as shown in Fig. 3a involves simultaneous inward displacement of four oxygen atoms in the tetramer. Therefore, the proton tunnelling is most likely coupled to the internal breathing mode of the tetramer. By applying the theory of phonon-assisted tunnelling, we can estimate the concerted tunnelling rate of four protons (Supplementary Fig. 7). The tip height dependence of the calculated tunnelling rate is qualitatively consistent with the experimental results shown in Fig. $2 c$, and full deuteration induces a substantial decrease of this tunnelling rate. A more quantitative comparison between theory and the experiments would require better knowledge of the asymmetry of the tunnelling barrier and the strength of coupling to the phonons ${ }^{25}$, which is beyond the scope of our present study.

To gain further insight into the tip-proton coupling, we plot the electron density differences (Fig. 3d-f) of the Cl-tip/tetramer/NaCl system (Fig. 3c) in a vertical plane (denoted by a dotted line in the topmost snapshot of Fig. 3a). DFT results show that the $\mathrm{Cl}$ atom at the tip apex is negatively charged, with a partial charge of about $0.4 e$. The nature of the local charge rearrangement in Fig. $3 \mathrm{~d}$ is characteristic of a weak electric interaction between $\mathrm{H}^{+}$and $\mathrm{Cl}^{\delta-}$ : with depletion (accumulation) of density on the $\mathrm{H}(\mathrm{Cl})$ atoms. Such an attractive interaction assists the proton transfer through the hydrogen bonds and thus suppresses the tunnelling barrier. When the tip approaches the tetramer, a greater electron accumulation and depletion are observed, indicating the increased electric interaction (Fig. 3e). However, when the tip is too close to the tetramer (Fig. 3f), Coulomb repulsion between $\mathrm{Cl}^{\delta-}$ and $\mathrm{O}^{2-}$ leads to considerable expansion of the tetramer, which rapidly increases the tunnelling barrier (Supplementary Fig. 8).

As well as the dependence on the tip height, the concerted proton tunnelling is rather sensitive to the lateral position of the $\mathrm{Cl}$ tip in the tetramer. The switching rates decrease by almost one order of magnitude in just $0.5 \AA$ as the tip moves from the centre to the edge of the tetramer along two axes of symmetry (Fig. 4). Such a fast decay of the switching rate can not be accounted for by the change of the tunnelling barrier as revealed by our tip-position dependent NEB calculations. Instead, it might arise from the collective nature of the proton tunnelling. When the tip is positioned near the centre of the tetramer, the four protons are equally coupled with the $\mathrm{Cl}$ anion and can be treated as a quantum quasiparticle, which moves in a fully correlated manner. However, slightly moving the tip off-centre leads to asymmetric coupling between the $\mathrm{Cl}$ anion and the four protons, which may break the degeneracy of the four hydrogen bonds and destroy the cooperativity of the four protons. In such a case, the quasiparticle tunnelling would be significantly suppressed, resulting in rapid quenching of the chirality switching. Similarly, destruction of the correlated collective proton tunnelling due to the formation of 


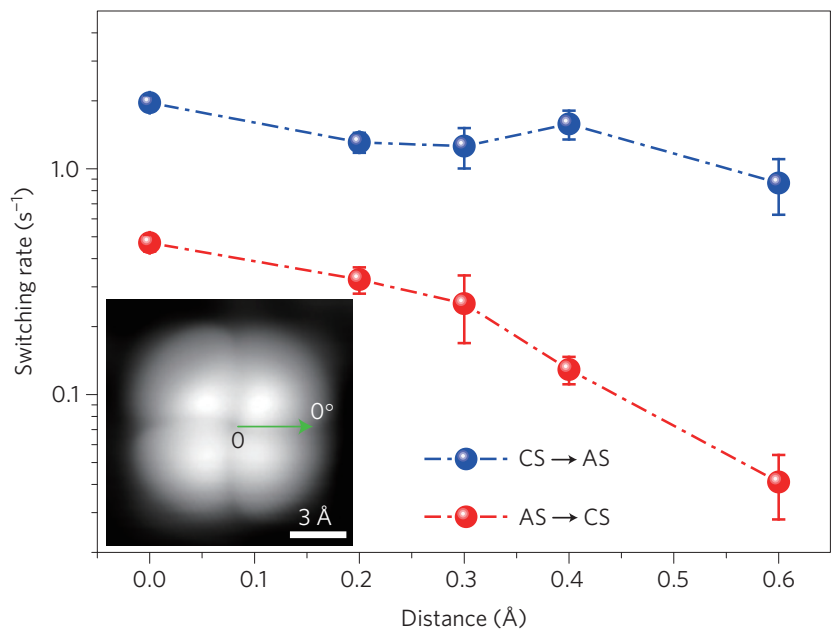

b

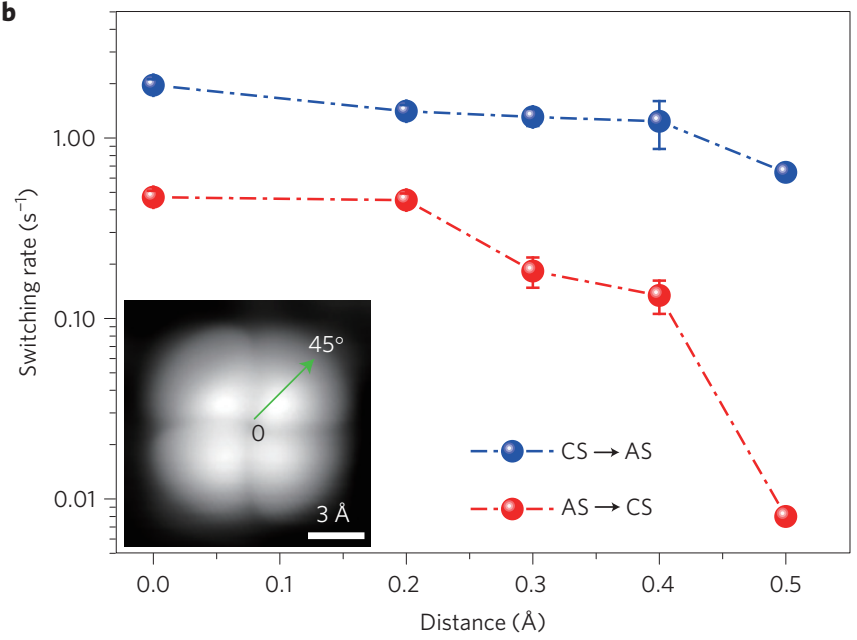

Figure 4 | Dependence of the switching rates on the lateral position of the tip. a, Switching rates as a function of tip position obtained by moving the tip along the $0^{\circ}$ direction away from the centre of the tetramer. $\mathbf{b}$, Same as $\mathbf{a}$ but along the $45^{\circ}$ direction. The green arrows in the insets denote the movement directions of the tip. The zero point of the tip position is set about $0.1 \AA$ away from the centre of the tetramer. The error bars represent the standard error. Sample bias: $5 \mathrm{mV}$. Tip height: $-265 \mathrm{pm}$ referenced to the gap set with $V=5 \mathrm{mV}$ and $I=5 \mathrm{pA}$.

inequivalent hydrogen bonds has been recently reported in partially deuterated hexagonal ice ${ }^{26}$.

Our real-space experimental observation provides strong evidence of the concerted proton tunnelling and reveals the important role of individual ions in influencing the correlated tunnelling process, which may shed new light on our understanding of the phase transition between different ice forms $\mathrm{s}^{7-9}$ as well as the hydrogen-bonding dynamics in aqueous salt solutions ${ }^{27}$. Furthermore, we demonstrated in this work the possibility of tuning the energy barrier for proton transfer by controlling the tip-proton coupling with a functionalized STM tip. By proper engineering of the tip apex and precise control of the tip height, it is possible to continuously suppress the reaction barrier such that the zero-point motion may surpass the barrier. Therefore, our methodology opens a new route for controlling the quantum states of the protons with atomic-scale precision.

\section{Methods}

The ultrahigh vacuum STM and the sample preparation are described elsewhere ${ }^{20}$. The $\mathrm{NaCl}(001)$ bilayer film was grown on a $\mathrm{Au}(111)$ surface at room temperature. The STM measurements were performed with electrochemically etched tungsten tips, which were repeatedly indented into the Au sample for tip shaping. The $\mathrm{Cl}$-terminated tip was obtained by approaching a bare tip to the $\mathrm{NaCl}(001)$ surface in close proximity $(V=5 \mathrm{mV}$ and $I=2 \mathrm{nA})$, followed by the application of a voltage pulse ( $2.5 \mathrm{~V}, 200 \mathrm{~ms}$; see Supplementary Fig. 1 for details). The controllable manipulation of $\mathrm{H}_{2} \mathrm{O}$ monomers to construct water tetramers was achieved with the Cl-terminated tip at a gap set with $V=10 \mathrm{mV}, I=150 \mathrm{pA}$. The deuterated tetramers, which consist of four $\mathrm{D}_{2} \mathrm{O}$ monomers, were constructed in separate experiments using a similar manipulation method. The time trace of the tunnelling current was recorded, with the feedback loop open, at $V=5 \mathrm{mV}, I=5 \mathrm{pA}$, after which different $z$ offsets were added to the tip height. The bias voltage refers to the sample voltage with respect to the tip. All the STM topographic images were obtained in the constant-current mode.

DFT calculations were performed using the Vienna ab-initio simulation package (VASP; ref. 28). Projector augmented wave pseudopotentials were used with a cutoff energy of $550 \mathrm{eV}$ for the expansion of the electronic wave functions ${ }^{29}$. A bilayer $\mathrm{NaCl}(001)$ slab with a lattice constant of $5.665 \AA$ was used according to the experimental conditions. The bottom layer of the $\mathrm{NaCl}$ was fixed during geometry relaxations. Van der Waals corrections for dispersion forces were considered using the van der Waals density functional scheme using the optB88-vdW method ${ }^{30}$. The Cl-terminated tip was modelled using a three-layer Au pyramid of a [111] cleaved face with a $\mathrm{Cl}$ atom attached at the end (see Fig. 3c). A Monkhorst-Pack grid with at least $8 \times 8 \times 1$ k-point sampling per $1 \times 1$ cell was used. Energy barriers and tetramer switching paths were determined using the cNEB method ${ }^{31}$. The effective energy barrier was calculated by subtracting from the original potential barrier the zero-point energy difference between the initial and transition states of the adsorbed water tetramer. The $\Gamma$-point harmonic vibration frequencies of the water tetramer were calculated using the finite displacement method, with the substrate fixed after the structural relaxation. The zero-point energy was estimated by a summation of half the vibrational energy of all $\Gamma$-point modes. The zero-point energy difference can then be written as $E(R)=(1 / 2)\left(\sum_{s} h v_{\text {init }}^{s}-\sum_{t} h v_{\text {tran }}^{t}\right)$, where $R$ is the equilibrium $\mathrm{O}-\mathrm{O}$ separation at different tip heights.

Received 31 July 2014; accepted 10 December 2014; published online 16 February 2015

\section{References}

1. Kreuer, K. D. Proton conductivity: Materials and applications. Chem. Mater. 8 , 610-641 (1996)

2. Benoit, M., Marx, D. \& Parrinello, M. Tunnelling and zero-point motion in high-pressure ice. Nature 392, 258-261 (1998).

3. Horiuchi, S. et al. Above-room-temperature ferroelectricity in a single-component molecular crystal. Nature 463, 789-793 (2010).

4. Frank, R. A. W. et al. A molecular switch and proton wire synchronize the active sites in thiamine enzymes. Science 306, 872-876 (2004).

5. Masgrau, L. et al. Atomic description of an enzyme reaction dominated by proton tunneling. Science 312, 237-241 (2006).

6. Brougham, D. F., Caciuffo, R. \& Horsewill, A. J. Coordinated proton tunneling in a cyclic network of four hydrogen bonds in the solid state. Nature 397, 241-243 (1999).

7. Bove, L. E., Klotz, S., Paciaroni, A. \& Sacchetti, F. Anomalous proton dynamics in ice at low temperature. Phys. Rev. Lett. 103, 165901 (2009).

8. Lin, L., Morrone, J. A. \& Car, R. Correlated tunneling in hydrogen bonds. J. Stat. Phys. 145, 365-384 (2011).

9. Drechsel-Grau, C. \& Marx, D. Quantum simulation of collective proton tunneling in hexagonal ice crystals. Phys. Rev. Lett. 112, 148302 (2014).

10. Gilli, G. \& Gilli, P. The Nature of the Hydrogen Bond (Oxford Univ. Press, 2009).

11. Garczarek, F. \& Gerwert, K. Functional waters in intraprotein proton transfer monitored by FTIR difference spectroscopy. Nature 439, 109-112 (2006).

12. Liljeroth, P., Repp, J. \& Meyer, G. Current-induced hydrogen tautomerization and conductance switching of naphthalocyanine molecules. Science 317, 1203-1206 (2007).

13. Kumagai, T. et al. $\mathrm{H}$-atom relay reactions in real space. Nature Mater. 11, 167-172 (2012).

14. Merte, L. R. et al. Water-mediated proton hopping on an iron oxide surface. Science 336, 889-893 (2012).

15. Auwärter, W. et al. A surface-anchored molecular four-level conductance switch based on single proton transfer. Nature Nanotech. 7, 41-46 (2012).

16. Kumagai, T. et al. Controlling intramolecular hydrogen transfer in a porphycene molecule with single atoms or molecules located nearby. Nature Chem. 6, 41-46 (2013)

17. Lawton, T. J. et al. Visualization of hydrogen bonding and associated chirality in methanol hexamers. Phys. Rev. Lett. 107, 256101 (2011).

18. Zhang, J. et al. Real-space identification of intermolecular bonding with atomic force microscopy. Science 342, 611-614 (2013).

19. Chiang, C. L. et al. Real-space imaging of molecular structure and chemical bonding by single-molecule inelastic tunneling probe. Science $\mathbf{3 4 4}$, 855-888 (2014). 
20. Guo, J. et al. Real-space imaging of interfacial water with submolecular resolution. Nature Mater. 13, 184-189 (2014).

21. Ho, W. Single-molecule chemistry. J. Chem. Phys. 117, 11033-11061 (2002).

22. Gawronski, H. et al. Manipulation and control of hydrogen bond dynamics in absorbed ice nanoclusters. Phys. Rev. Lett. 101, 136102 (2008).

23. Lauhon, L. J. \& Ho, W. Direct observation of the quantum tunneling of single hydrogen atoms with a scanning tunneling microscope. Phys. Rev. Lett. 85, 4566-4569 (2000).

24. Leggett, A. J. et al. Dynamics of the dissipative two-state system. Rev. Mod. Phys. 59, 1-85 (1987).

25. Horsewill, A. J. Quantum tunnelling in the hydrogen bond. Prog. Nucl. Magn. Reson. Spectrosc. 52, 170-196 (2008).

26. Drechsel-Grau, C. \& Marx, D. Exceptional isotopic-substitution effect: Breakdown of collective proton tunneling in hexagonal ice due to partial deuteration. Angew. Chem. 126, 11117-11120 (2014).

27. Ding, Y., Hassanali, A. A. \& Parrinello, M. Anomalous water diffusion in salt solutions. Proc. Natl Acad. Sci. USA 111, 3310-3315 (2014).

28. Kresse, G. \& Hafner, J. Ab initio molecular dynamics for liquid metals. Phys. Rev. B 47, 558-561 (1993).

29. Kresse, G. \& Joubert, D. From ultrasoft pseudopotentials to the projector augmented-wave method. Phys. Rev. B 59, 1758-1775 (1999).

30. Klimeš, J., Bowler, D. R. \& Michaelides, A. Van der Waals density functionals applied to solids. Phys. Rev. B 83, 195131 (2011).

31. Henkelman, G., Uberuaga, B. P. \& Jónsson, H. A climbing image nudged elastic band method for finding saddle points and minimum energy paths. J. Chem. Phys. 113, 9901-9904 (2000).

\section{Acknowledgements}

This work was supported by the National Basic Research Programs of China under Grant Nos 2012CB921303, 2012CB921304 and 2013CB934600, the National Science Foundation of China under Grant Nos 11104004, 11274012, 91021007 91321309, 11290162/A040106 and 11275008. Y.J. acknowledges support from the National Program for Support of Top-notch Young Professionals. We are grateful for the computational resources provided by the supercomputer TianHe-1A in Tianjin, China. We thank Z. H. Cheng, A. H. Castro Neto, M. F. Crommie and A. Hodgson for enlightening discussions.

\section{Author contributions}

Y.J. and E-G.W. designed and supervised the project. X.M., J.G. and Y.J. performed the STM measurements. J.C. and X-Z.L. carried out the DFT calculations. J.P., J.G., X.M., Z.W., and Y.J. analysed the data. J-R.S. contributed to the interpretation of the data. Y.J. and X.M. wrote the manuscript with J.G., J.P., J.C., X-Z.L. and E-G.W. The manuscript reflects the contributions of all authors.

\section{Additional information}

Supplementary information is available in the online version of the paper. Reprints and permissions information is available online at www.nature.com/reprints.

Correspondence and requests for materials should be addressed to E-G.W. or Y.J.

\section{Competing financial interests}

The authors declare no competing financial interests. 